\title{
Characteristics and models of human symmetry detection
}

\author{
Johan Wagemans
}

\begin{abstract}
Symmetry is everywhere - in natural objects, from crystals to living organisms, in manufactured articles of many kinds, and in art works from all cultures. Symmetry is a salient visual property that is detected efficiently and rapidly by the human visual system. In this paper, several decades of experimental research on human symmetry detection are reviewed. By examining the effects of several factors on symmetry detection, this research has revealed some important characteristics of how humans perceive symmetry. These characteristics constrain the general principles of putative underlying mechanisms and models of human symmetry detection. For example, the orientation of the axis of symmetry and its location in the visual field have effects that suggest that the bilateral symmetry of the visual system at cortical levels of the brain might partly determine the salience of vertical mirror symmetry. At the same time, there is a surprisingly high degree of flexibility and robustness that remains to be explained. Thus, symmetry provides a major challenge to model human flexibility and efficiency within the constraints of the biology of the visual system.
\end{abstract}

A A the shapes in Fig. $1 \mathrm{~A}$ and $1 \mathrm{~B}$ are obviously not random. They have a simple, regular structure which makes them 'good Gestalts'. Similarly, the dots in Fig. IC, E, and F seem to be grouped together in regular configurations that clearly differ from random-dot patterns such as the one in Fig. 1D. The same is true for the pattern shown in Fig. 1G, although more careful inspection will reveal that it is not perfectly regular but slightly perturbed. The converse holds for Fig, 1H, which might appear random at first sight, despite the fact that it shows a similar pattern as in Fig. 1C but viewed from a different angle.

Why is it that our visual system seems so well attuned to regularity? How do we detect the regularity in such patterns? Why are some structures easier to see than others? How much of this is based on the special status of faces for visual perception? In this paper, several decades of experimental work are reviewed that were inspired by phenomenological observations, such as those made in relation to Fig. 1 (Ref. 1). As a result of this research effort, much is known about the effects of several factors on human detection of symmetry. These effects help to specify the characteristics of human symmetry detection, as well as the general principles of putative underlying mechanisms. Although the rich collection of empirical data has not yet converged upon one universally accepted theory or model of human symmetry detection, the data do suggest important constraints within which the proposed mechanisms have to operate. The mod- elling of symmetry detection presents challenges that are typical of most work in cognitive science.

Is mirror symmetry special?

What is the structure or regularity that is shared by all the patterns in Fig. 1 except pattern 1D? What do they have in common for them to be called 'symmetric'? In other words, what is symmetry? Informally, symmetry means selfsimilarity under a class of transformations, usually restricted to the Euclidean transformations in the plane, namely, translations, rotations and reflections. For example, the shapes in Fig. $1 \mathrm{~A}$ and $\mathrm{B}$ can be reflected about a straight axis and the resulting shape will be indistinguishable from the original. The orientation of the axis differs between them (i.e. vertical for Fig. 1A, oblique for 1B) but the identity under a reflection makes both of them mirror-symmetric or bilaterally symmetric. In Fig. 1C, E and F, a random-dot pattern was taken and then reflected about a vertical axis (1C), translated across a small horizontal distance (1E), or rotated by $180^{\circ}(1 \mathrm{~F})$. The resulting symmetries are called reflectional, translational and rotational, respectively.

If all of these structures are symmetric, why does mirror symmetry seem special? Most people will agree that the regularity or structure is more salient in Fig. 1C than in Fig. $1 \mathrm{E}$ and $1 \mathrm{~F}$. The detection of symmetry created by translation or rotation seems to require scrutiny, whereas perception of mirror symmetry (i.e. created by reflection) is effortless, 

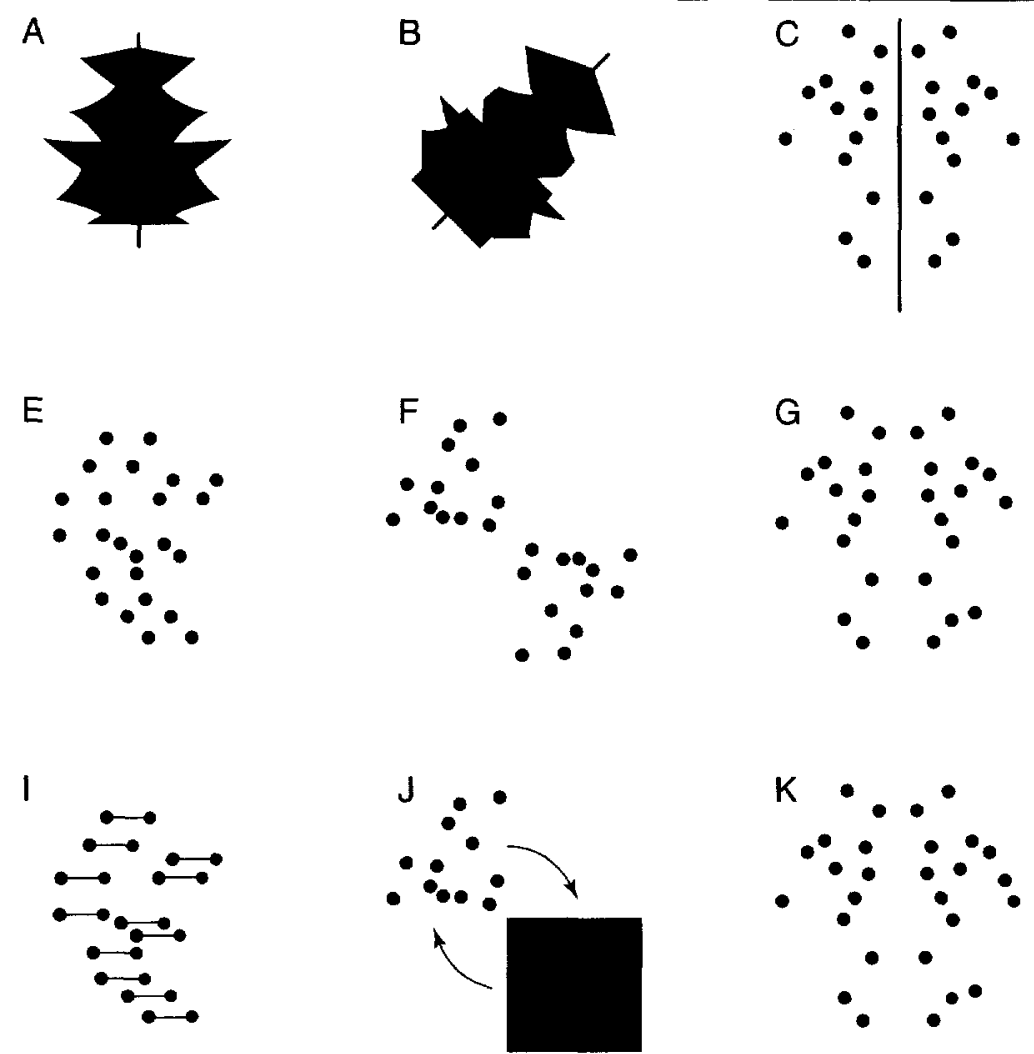

$\mathrm{L}$

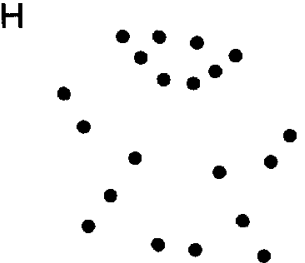

Fig. 1 Examples of typical stimuli used in symmetry detection experiments. (A) A polygon with mirror symmetry about a vertical axis (indicated by solid line). (B) A polygon with mirror symmetry about a diagonal axis (indicated by solid line). (C) A random-dot pattern with mirror symmetry about a vertical axis (indicated by solid line). (D) A completely random dot pattern. (E) A random-dot pattern with translational symmetry. The translation vector is indicated in (I) underneath. (F) A random-dot pattern with a rotational symmetry. The $180^{\circ}$ rotation is indicated in $(J)$ underneath. (G) A random-dot pattern with perturbed mirror symmetry. The perturbations are indicated in (K) underneath. (H) A random-dot pattern with skewed mirror symmetry. The skewing is indicated in (L) underneath.

rapid and spontaneous ${ }^{2}$. This preference for mirror symmetry is shown in researchers' preoccupation with this type of symmetry and it has also been corroborated experimentally, when compared with translational symmetry ${ }^{3,4}$, with rotational symmetry ${ }^{5,6}$, or with both within the same study ${ }^{7.8}$. Some theorists have attempted to explain the preference for mirror symmetry by referring to the mirror symmetry of the brain itself ${ }^{1,2,9}$. Some evidence related to this hypothesis is discussed below.

Before that, another important question arises: is it really true that reflectional symmerry can be detected preattentively? If one believes that a reduction in presentation time is sufficient to tap preattentive processes, this question can be answered positively. Mirror symmetry can be perceived in a wide variety of stimuli, from simple dot patterns to complex abstract art displays, when flashed very briefly (for 10-100 ms) ${ }^{10-12}$. However, in all of these studies, attention is involved in that subjects are explicitly instructed to detect symmetry. Recent research has shown that many of the phenomena of grouping and perceptual organization studied by Gestalt psychologists do not occur when subjects' attention is not directed to the stimulus array and the task at hand ${ }^{13,14}$

Fortunately, there is also evidence that mirror symmetry is picked up and used, even when the task does not require subjects to do so. For example, visual search for a simple oriented line segment was shown to be affected by the symmetry of the background elements ${ }^{15}$. More interestingly, a patient with brain damage centred on the right parietal lobe, causing left visual neglect (i.e. so-called 'blindness' for everything to the left of fixation), still organized ambiguous figure-ground displays with symmetric shapes as figures, just as normal perceivers do, although he was unable to detect the symmetry when asked explicitly ${ }^{16}$. In other words, at some preattentive level in the visual system, the symmetry of the shapes was picked up and it affected figure-ground organization, despite the fact that the mental representation of this visual attribute of the shapes could not be accessed consciously.

\section{Major factors influencing detectability}

Axis orientation

Casual observations suggest that mirror symmetry is easier to see when the axis is oriented vertically (Fig. 1A) than with a horizontal or a diagonal (Fig. 1B) axis. Results from several studies examining the relative difficulties of detecting mirror symmetry in the four cardinal orientations (vertical, horizontal and the two diagonals) have confirmed these observations ${ }^{17-21}$. Only a few studies have tested other oblique orientations in addition to the main diagonals. The results here suggest the following detectability ordering (where ' $>$ ' means better performance): vertical > horizontal $>$ near vertical $>$ diagonal $>$ near horizontal $>$ the other obliques $^{17-19}$. This pattern is, however, incongruent with 


\section{Box 1. Characteristics of human symmetry detection}

1 The human visual system has a preference for mirror symmetry, but that does not mean that other regularities, such as translarional or rotational symmetries, cannot be detected. 2 Mirror symmetry can be detected preattentively, but that does not mean that attentional or scanning strategies play no role at all.

3 Mirror symmetry is easier to detect when the axis is oriented vertically than horizontally or obliquely, but the axisorientation effect does not simply mimic the neural sensitivities of orientation-selective cells.

4 Mirror symmetry is easier to detect when the axis passes through the point of fixation, but that does not mean that it remains undetected at more peripheral locations.

5 Detection of mirror symmetry depends most strongly on elements close to the axis, but the edge of a pattern is also important and symmetry can be detected without any elements close to the axis.

6 Human symmetry detection is both robust and sensitive to small perturbations.

7 The identity and location of individual elements is less important for high-density patterns than for low-density patterns.

the neurophysiological evidence on the oblique effect (i.e. the disadvantage of oblique orientations relative to vertical and horizontal), which indicates that the sensitivity of the visual system to orientation decreases with larger deviations from vertical and horizontal ${ }^{20}$.

Moreover, recent research has clearly demonstrated that the detectability of mirror symmetry at a certain axis depends on the frequencies of different orientations within a block of trials ${ }^{19}$. For example, when the 16 orientations tested were vertical, horizontal, the two diagonals, and $5^{\circ}$, $10^{\circ}$ and $15^{\circ}$ deviations from the diagonals (clockwise and counterclockwise), performance was superior for the diagonal symmetries, not vertical or horizontal symmetry. These results imply that the orientation effects on symmetry detection are not completely determined by the fixed neural architecture of the visual system but can be modulated to a large extent by the subjects' scanning or attentional strategies $^{18,19,21}$.

\section{Location in the visual field}

If the salience of vertical symmetry were to depend on the bilateral symmetry of the brain, symmetric projection of retinal patterns to the cortical visual system would be essential for efficient symmetry detection. Although mirror symmetry is indeed easier to detect when the axis of reflection passes through the point of fixation ${ }^{17,22}$, central presentation is much less critical for closed patterns, such as Fig. $1 \mathrm{~A}$ and $1 \mathrm{~B}$, than for dot patterns, such as Fig. 1C. This distinction suggests that symmetry detection might operate at two levels: for patterns with low spatial frequencies (e.g. Fig. 1A and 1B), the symmetric relations can be extracted globally, whereas a point-by-point comparison seems required for patterns with high spatial frequencies (e.g. Fig. 1C-H ${ }^{2}$.

\section{Local versus global processing}

Although all elements in symmetric patterns need corresponding elements at the symmetric position on the other side of the axis, the visual system need not compare all of them to signal the presence of mirror symmetry. Three sources of evidence support this.

First, several studies have demonstrated that the elements closest to the axis contribute most to the symmetry percept ${ }^{3,17,23,24}$. However, even without corresponding internal features in the immediate neighbourhood of the axis, bilateral symmetry can still be perceived ${ }^{10}$ and elements near the edge of the pattern have been shown to play an important additional role ${ }^{17,24}$.

Second, small perturbations of many kinds often go unnoticed at first sight. For example, human faces are never perfectly symmetrical. This becomes evident, however, only when pictures of faces are cut vertically into two halves, which are then reflected to create two perfectly symmetric pictures. The faces in these pictures clearly differ from each other as well as from the original. This suggests that mirror symmetry is a canonical property that tends to be exaggerated by the visual system ${ }^{25,26}$. However, in animals where deviations from perfect symmetry can be correlated with gene deficiencies, it might be important to be able to detect those small perturbations as well ${ }^{27,28}$. Somewhat surprisingly, psychophysical research supports both of these apparently conflicting intuitions ${ }^{29}$. On the one hand, patterns with only $30 \%$ symmetric dot-pairs are still categorized as symmetric when they have to be discriminated from random distractors. On the other hand, small perturbations from perfect symmetry are detected in a perfect versus imperfect symmetry discrimination task. Thus, symmetry detection is both robust and sensitive to perturbation ${ }^{17,29}$.

Third, in symmetrical displays consisting of oriented line elements, the spatial grouping (e.g. clustering) of the line segments has a larger effect on the detectability of the symmetry than their individual identities (e.g. their orientation relative to the axis of symmetry $)^{12,30}$. These and other results suggest that the perception of symmetry might be nothing more than the conscious concomitant of the output of the normal filtering operations executed in parallel on a symmetrical display, which would make the locations of large-scale tokens (e.g. blobs) available before the figural identity of the elements ${ }^{6,12,17,21,29,31}$. This idea has been incorporated into many different theorerical accounts or computational models of symmetry detection.

\section{Theoretical accounts and computational models of} putative mechanisms for human symmetry detection Several decades of research into human symmetry detection have revealed many of its basic characteristics (Box 1). These constitute a surprisingly complicated pattern to capture theoretically. One important question is whether a single mechanism can produce such a diversity of characteristics or, alternatively, whether a multitude of mechanisms exist for detecting symmetry in different conditions. For example, the salience of vertical symmetry at fixation has led some people to suggest that human symmetry detection is based on a point-by-point matching process between symmetrically opposite loci in each cortical hemisphere that 
would be mediated by fibres crossing over through the corpus callosum ${ }^{1,2,9,32,33}$. Although there is some empirical support for this hypothesis, it would necessarily imply the involvement of other mechanisms for detecting mirror symmetry at non-vertical orientations, or at locations in the visual field away from fixation. Detailed examination of psychophysical sensitivity functions of mirror symmetry detection has led other authors to suggest the existence of at least five distinct mechanisms for the detection of mirror symmetry only ${ }^{34}$. At the other extreme are models that attempt to capture all sorts of regularity detection, including reflectional, translational and rotational symmetry, under one single theoretical umbrella, either in a process model ${ }^{8}$, or in a mathematical representation of pattern regularity ${ }^{35}$.

\section{Theoretical accounts}

In the 1970 s and early 1980 s, several theoretical accounts of human symmetry detection were proposed that seemed to justify some of its basic characteristics but that were not detailed enough to elaborate algorithms that could be computer-simulated and tested. Firstly, for example, Palmer and Hemenway' proposed a dual-process model consisting of a selection-evaluation cycle. A potential axis of symmetry would first be selected by a rapid, but crude, analysis of symmetry in all orientations simultaneously. By establishing a perceptual reference frame in the appropriate orientation, a detailed evaluation would then be performed by explicit, pointwise comparison of the two pattern halves. This model can explain many of the well-established facts about symmetry detection. For example, that multiple symmetries (i.e. created by reflection about more than one axis) are more salient than single symmetries can be explained by assuming a variable order of selection, which, on average, leads to faster axis selection when there are many axes to choose from. However, this model does not explain why the multiple symmetry advantage remains even when only symmetry about a specific axis has to be reported.

Secondly, Barlow and Reeves ${ }^{17}$ proposed that the only thing the visual system does in detecting symmetry is to compare dot densities measured in relatively large areas placed symmetrically about the putative axis of symmetry. Although this seems to be an operation that could be performed quite easily by visual neurons with fixed receptive fields, it is not at all clear how the visual system could make all the comparisons required for each different position and orientation of the axis without confusion. Moreover, this proposed mechanism cannot explain symmetry detection in patterns in which density has been made homogeneous, such as in Fig. 1C-H (Ref. 36).

Thirdly, Jenkins ${ }^{37}$ proposed that the detection of bilateral symmetry in dense dot textures involves three component processes: (1) a process that detects that the imaginary lines joining pairs of symmetrically positioned dots have uniform orientations; (2) a process that fuses the most salient point-pairs around the axis into a larger feature; and (3) a process that determines whether that larger-scale feature is symmetrical, probably by determining whether the midpoints between the corresponding points are perfectly collinear. Assuming that each of these component processes responds differently to variations in axis orientation, this model can explain a large number of experimental data without postulating the existence of a symmetrical neural organization centred about the fovea. However, if establishing orientational uniformity and midpoint collinearity are indeed the major processes underlying symmetry detection, it is not clear why symmetries such as those in Fig. $1 \mathrm{H}$ are more difficult to detect, because all the point-pairs in such patterns have the same orientation and collinear midpoints just as in Fig. 1C (see also Box 2).

Thus, each of these three process models, representative of theoretical accounts of human symmetry detection formulated in the 1970 s and early 1980 s, has its own problems in addition to the fact that they are too general to be implemented computationally. As in many other areas of cognitive science, truly computational models came only later.

\section{Computational models}

One of the first explicit models of symmetry detection ${ }^{8.38}$, including computer simulations of psychophysical results with an implemented version of the model, started from the observed failures of Jenkins' account ${ }^{37}$. Whenever perfectly bilateral symmetry is viewed from a non-orthogonal viewpoint, the actual projection on the retina is skewed as in Fig. $1 \mathrm{H}$. This means that corresponding elements are not at orthogonal positions across the axis, but at an orientation that differs from $90^{\circ}$, with an offset (i.e. the skew angle) determined by the viewing position. An extensive series of experiments with these skewed symmetries demonstrated that symmetry becomes harder to detect with increasing skew angle, even when the point-pairs are oriented horizontally or have midpoints that are aligned vertically, as in the simple case of perfect vertical mirror symmetry ${ }^{8,18,38,39}$. What distinguishes skewed from non-skewed symmetries has, therefore, nothing to do with first-order regularities defined on point-pairs (such as orientational uniformity and midpoint collinearity; Fig. $2 \mathrm{~A}$ ). The critical difference is the presence of regular second-order structures in perfect mirror-symmetric patterns (such as symmetric trapezoids; Fig. 2A) which are destroyed by skewing (Fig. 2B).

In our own model of symmetry detection ${ }^{8.38}$, it is assumed that these regular higher-order structures facilitate the propagation of local pairwise groupings, a process which was called 'bootstrapping'. The basic idea of the bootstrap model is that symmetric trapezoids specify a reference frame that suggests a unique direction within which other correspondences are much more likely to be found. In other words, the initial randomness in pairing of elements in a pattern within some local neighbourhood converges more rapidly and more efficiently to a coherent global structure (for further details, see Box 2).

This bootstrap model was additionally tested by comparing the effects of skewing in single, double and fourfold symmetries $^{38}$. As expected, skewing was only detrimental to the degree that it destroyed the higher-order structures. Even with other types of symmetry, such as those created by translation and rotation, it was shown that the detection of symmetry is easy when the pairwise correspondences are supported by regular higher-order structures and more difficult when only lower-order structure is available ${ }^{8}$. In the same study, a possible implementation of the bootstrap 


\section{Box 2. The bootstrap model}

The 'bootstrap model' is a process model that starts from the available information in a dot pattern (i.e. the locations of the dots). Dots are grouped in pairs using a virtual line to establish a connection. Initially, pairwise groupings are random although the grouping process has some built-in preferences (e.g. it starts in the middle of a pattern and it has a preference for short, horizontal virtual lines). Virtual lines connecting symmetrically positioned dots have uniform orientations and collinear midpoints, two properties that were found to be useful in symmetry detection. In perfect mirror symmetry, a pair of virtual lines connecting symmetrically positioned dots also establishes a virtual quadrangle with correlated angles (called a correlation quadrangle', Fig. 2A-C). These correlation quadrangles establish a local reference frame that suggests a unique direction within which other correspondences are much more likely to be found (i.e. the axis of symmetry). They facilitate the propagation of local pairwise groupings throughout the whole pattern so that the initial randomness in pairing of elements within some local neighbourhood becomes systematic much more easily, establishing a coherent global structure more rapidly and more efficiently. This automatic spreading-out of correspondences is called 'bootstrapping'.

When mirror-symmetric dot patterns are viewed from aside, one has so-called 'skewed symmetry' (Fig. 2D-F). As a result of skewing, the first-order regularities of the virtual lines (i.e. orientational uniformity and midpoint collinearity) are preserved but the second-order regularities of the virtual quadrangles are destroyed (i.e. the angles in the virtual quadrangles are no longer pairwise correlated). Thus, bootstrapping is no longer possible. These properties of the bootstrap model provide explanations of the superior detectability of orthofrontal mirror symmetry compared with skewed symmetry, ${ }^{\text {b,c }}$, of double mirror symmetry compared with single mirror symmetry ${ }^{b, d e}$, and of the smaller effect of skewing with multiple symmetries ${ }^{b}$.

The implemented version of the model consists of two basic parts. The first is a function that captures the cost of grouping. It has two components, one for the first-order regularity (quantified as the deviation from parallelism) and one for the second-order regularity (quantified as the deviation from pairwise correlated angles). The second basic part of the bootstrap model is an algorithm that optimizes the cost function using a procedure that is similar to simulated annealing. It makes local groupings that are initially random converge to a stable organization that reflects the true global structure of a pattern. It has one free parameter, the initial 'temperature', that determines how easily initial groupings can change. While the grouping process continues (in several iterative steps), the temperature is decreased so that the chance that groupings are destroyed also decreases.

\section{References}

a Jenkins, B. (1983) Component processes in the perception of bilaterally symmetric dot textures Percept. Psychophys. 34, 433-440

b Wagemans, J., Van Gool, L. and d'Ydewalle, G. (1991) Detection of symmetry in tachistoscopically presented dot patterns: Effects of multiple axes and skewing Percept. Psychophys. 50, 413-427

c Wagemans, J., Van Gool, L. and d'Ydewalle, G. (1992) Orientational effects and component processes in symmetry detection Q. J. Exp. Psychol. 44A, 475-508

d Palmer, S.E. and Hemenway, K. (1978) Orientation and symmetry: Effects of multiple, rotational, and near symmetries J. Exp. Psychol. Human Percept. Perform. 4,691-702

e Royer, F.L. (1981) Detection of symmetry J. Exp. Psychol. Human Percept. Perform. 7, 1186-1210

$f$ Kirkpatrick, S., Gelatt, C.D. and Vecchi, M.P. (1983) Optimization by simulated annealing Science 220, 671-680 model was proposed, and good qualitative agreement was obtained between the experimental data and the simulation results.

The specific details of the bootstrap model in its current form can be applied only to high-spatial-frequency, lowdensity patterns consisting of discrete elements (preferably dots). However, a model along the same lines could be developed for low-spatial-frequency patterns, such as dense dot textures or closed figures, by assuming that the processes operate on the centroids of blobs or the vertices of polygons. The centroids of blobs could be obtained from a filter model like the one proposed by Dakin and Watt ${ }^{40}$.

As indicated earlier, several findings about human symmetry detection suggest that spatial filtering mechanisms are involved. For example, the area around the midline of bilaterally symmetric patterns contributes more to the perception of symmetry than other areas, and position-based grouping of individual elements into larger clusters is often more important than their featural identity. It is Dakin and Watt's major contribution to have been the first to capture this into an explicit filter mode ${ }^{40}$. It comprises two parts, a filter to obtain the major clusters or blobs, and a mechanism to compare the locations of the blobs along the putative axis of symmetry. The filters work either homogeneously in all directions or with a bias in a certain orientation (e.g. or- thogonal to the axis of symmetry); the matching scheme was either a perfect, but computationally very demanding, correlation measure or an approximate, but neurally more plausible, alignment measure. Combinations of these filters and matching schemes thus provided four possible models. Several sets of well-established findings $s^{17,37}$ were simulated to test the plausibility of each of the four models as mechanisms for human symmetry detection. Only the performance of the model with oriented filters in combination with the alignment matching scheme was consistent with human performance in all conditions.

A final model, which was also implemented computationally and tested against human performance, is a three-stage computational model for the detection of mirror symmetry in dense texture-like patterns with oriented line segments ${ }^{30}$. In the first stage, primitive elements with similar features (e.g. length and orientation) are grouped into a smaller number of clusters. In the second stage, pairs of clusters are compared to establish local symmetry axes. In the third and final stage, the local symmetries are aggregated to form global symmetry relations. It should be pointed out that the first and final stages are useful in many more visual tasks than merely symmetry detection. Another interesting aspect of this model is that it seems to integrate aspects of both earlier models. Thus, although the specific algorithms 
differ, the computations performed in the first and second stage are similar to those performed by both components of Dakin and Watt's filter model ${ }^{40}$, while the computations of the second and third stage are similar to those performed by both components of our own bootstrap model $^{8.38}$. An extensive discussion of all relationships between these three models is beyond the scope of the present review. However, it should be clear that they need not be considered as mutually exclusive approaches.

\section{Conclusions}

Human symmetry detection appears to be a benchmark case for cognitive science. Extensive experimental research has demonstrated convincingly that it is a remarkably efficient and versatile process, like many other perceptual and cognitive processes produced by the human mind. Computational models have begun to tackle the challenge of unravelling the details of its mechanisms in ways that attempt to integrate three mutually constraining requirements: to do justice to as many basic characteristics as possible of the mechanisms that have been discovered by experimental psychology, to formulate algorithms in enough detail to allow computer implementation, and to take into consideration as much as possible of what we know about the visual system as implemented in the human brain.

\section{Acknowledgements}

The author was supported by grants G.0210.97N and G.0130.98 from the Research Program of the Fund for Scientific Research - Flanders (Belgium) (FWO). Useful comments on a previous version have been provided by Paul Locher and three anonymous reviewers.

\section{References}

1 Mach, E. (1959) The Analysis of Sensations (originally published in German in 1886), Dover

2 Julesz, B. (1971) Foundations of Cyclopean Perception, University of Chicago Press

3 Bruce, V.G. and Morgan, M.J. (1975) Violations of symmetry and repetition in visual patterns Perception 4, 239-249

4 Baylis, G.C. and Driver, J. (1994) Parallel computation of symmetry but not repetition within single visual shapes Visual Cognition 1, 377-400

5 Palmer, S.E. and Hemenway, K. (1978) Orientation and symmetry: effects of multiple, rotational, and near symmetries J. Exp. Psychol. Hum. Percept. Perform. 4, 691-702

6 Royer, F.L. (1981) Detection of symmetry J. Exp. Psychol. Hum. Percept. Perform. 7, 1186-1210

7 Kahn, J.I. and Foster, D.H. (1986) Horizontal-vertical structure in the visual comparison of rigidly transformed patterns J. Exp. Psychol. Hum. Percept. Perform. 12, 422-433

8 Wagemans, J. et al. (1993) Higher-order structure in regularity detection Vis. Res. 33, 1067-1088

9 Herbert, A.M. and Humphrey, G.K. (1996) Bilateral symmetry detection: testing a 'callosal' hypothesis Perception 25, 463-480

10 Carmody, D.P., Nodine, C.F. and Locher, P.J. (1977) Global detection of symmetry Percept. Motor Skills 45, 1267-1273
B

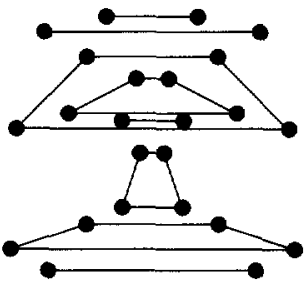

$E$



C
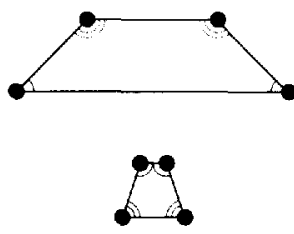

F
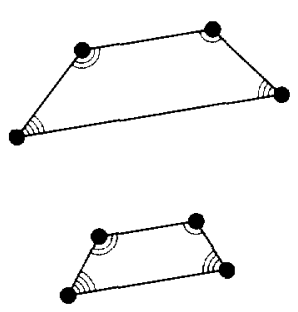

\section{Outstanding questions}

- What does it mean to say that mirror symmetry is detected preattentively? Clearly, attentional strategies sometimes play a role, as in determining the orientation effect and in detecting small perturbations. Is mirror symmetry strong enough to capture attention also when it is present in the periphery of a crowded scene? If so, it could be useful for perceptual processes preceding object identification, such as segmentation and eye movement guidance.

- Detection of vertical mirror symmetry at fixation is clearly superior to detection of non-vertical mirror symmetries away from fixation or other types of symmetry. To what extent does this imply a role for the bilateral symmetry of the visual cortex? What is the ecological significance of vertical mirror symmetry? Two-dimensional bilateral symmetry does not seem to be more common than other regularities, especially when not restricted to orthogonal viewing conditions. Perhaps it has become important because of the role of facial expression in social relationships.

- How can we explain that symmetry detection is, at the same time, robust and sensitive to small perturbations? Are two separate processes involved, or is it a matter of tuning into different aspects of the same perceptual representation?

- Grouping of neighbouring elements sharing similar properties clearly plays a role in symmetry detection but symmetry itself has been identified by Gestalt psychology as a grouping factor. Is this a case of mutual reinforcement of different grouping principles (i.e. proximity, similarity, symmetry), which is typical for Gestalt phenomena? Similarly, the essential role of elements close to the axis suggests some sort of interaction between local position-based grouping and more global symmetry detection. Is 'bootstrapping' the best way to capture this parallel and interactive way of processing?

- A large number of factors have been shown to influence the detectability of symmetry, but symmetry detection nevertheless appears to be a remarkably robust and versatile process. Do we have to capture this paradox theoretically by one single mechanism that can operate in a large number of conditions, or by a large number of highly specialized symmetry detection mechanisms for each of these conditions? 
11 Locher, P.J. and Nodine, C.F. (1989) The perceptual value of symmetry Comp. Math. Appl. 17, 475-484

12 Locher, P.J. and Wagemans, J. (1993) The effects of element type and spatial grouping on symmetry detection Perception 22, 565-587

13 Mack, A. et al. (1992) Perceptual organization and attention Cognit. Psychol. 24, 475-501

14 Rock, l. et al. (1992) Perception without attention: results of a new method Cognit. Psychoi. 24, 502-534

15 Wolfe, J.M. and Friedman-Hill, S.R. (1992) On the role of symmetry in visual search Psychol. Sci. 3, 194-198

16 Driver, J., Baylis, G.C. and Rafal, R. (1992) Preserved figure-ground segregation and symmetry detection in visual neglect Nature 360 , $73-75$

17 Barlow, H.B. and Reeves, B.C. (1979) The versatility and absolute efficiency of detecting mirror symmetry in random dot displays Vis. Res. 19, 783-793

18 Wagemans, J., Van Gool, L. and d'Ydewalle, G. (1992) Orientational effects and component processes in symmetry detection Q. J. Exp. Psychol. 44A, 475-508

19 Wenderoth, P. (1994) The salience of vertical symmetry Perception 23 , 221-236

20 Appelle, S. (1972) Perception and discrimination as a function of stimulus orientation: The 'oblique effect' in man and animals Psychol. Bull. 78, 266-278

21 Pashler, H. (1990) Coordinate frame for symmetry detection and object recognition J. Exp. Psychol. Hum. Percept. Perform. 16, 150-163

22 Saarinen, J. (1988) Detection of mirror symmetry in random dot patterns at different eccentricities Vis. Res. 28, 755-759

23 Jenkins, B. (1982) Redundancy in the perception of bilateral symmetry in dot textures Percept. Psychophys. 32, 171-177

24 Wenderoth, P. (1995) The role of pattern outline in bilateral symmetry detection with briefly flashed dot patterns Spatial Vis. 9, 57-77

25 Freyd, J.J. and Tversky, B. (1984) Force of symmetry in form perception Am. J. Psychol. 97, 109-126
26 McBeath, M.K., Schiano, D.J. and Tversky, B. (1997) Three-dimensional bilateral symmetry bias in judgments of figural identity and orientation Psychol. Sci. 8, 217-223

27 Meller, A.P. (1992) Fernale swallow preference for symmetrical male sexual ornaments Nature 357, 238-240

28 Swaddle, J.P. and Cuthill, I.C. (1993) Preference for symmetric males by female zebra finches Nature 367, 165-166

29 Wagemans, J. (1995) Detection of visual symmetries Spatial Vis. 9 9-32

30 Labonté, F. et al. (1995) A model for global symmetry detection in dense images Spatial Vis. 9, 33-55

31 Osorio, D. (1996) Symmetry detection by categorization of spatial phase: a model Proc. $R$. Soc. London Ser. B 263, 105-110

32 Corballis, M.C. and Beale, I.L. (1976) The Psychology of Left and Right Eribaum

33 Braitenberg, V. (1990) Reading the structure of brains Network 1, 1-11

34 Tyler, C.W., Hardage, L. and Miller, R.T. (1995) Multiple mechanisms for the detection of mirror symmetry Spatial Vis. 9, 79-100

35 Van der Helm, P.A. and Leeuwenberg, E.L.J. (1996) Goodness of visual regularities: a nontransformational approach Psychol. Rev. 103 429-456

36 Wenderoth, P. (1996) The effect of dot pattern parameters and constraints on the relative salience of vertical bilateral symmetry Vis. Res. 36, 2311 2320

37 Jenkins, B. (1983) Component processes in the perception of bilaterally symmetric dot textures Percept. Psychophys. 34, 433-440

38 Wagemans، J., Van Gool, L. and d'Ydewalle, G. (1991) Detection of symmetry in tachistoscopically presented dot patterns: effects of multiple axes and skewing Percept. Psychophys. 50, 413-427

39 Wagemans, J. (1993) Skewed symmetry: a nonaccidental property used to perceive visual forms J. Exp. Psychol. Hum. Percept. Perform. 19, 364-380

40 Dakin, S.C. and Watt, R.J. (1994) Detection of bilateral symmetry using spatial filters Spatial Vis. 8, 393-413

\section{Finally, the end of the first year of Trends in Cognitive Sciences.}

The first year of the existence of any journal is a gradual learning period during which the journal relies upon the goodwill, cooperation and support of all the people who contribute to its production and distribution. Trends in Cognitive Sciences was no exception. We would therefore like to acknowledge and thank the authors and the referees for their excellent contributions. Their extremely rapid and good-natured responses to our requests were a vital part of the first year of the journal. We would also like to thank the Advisory Editorial Board for their valuable advice, suggestions and enthusiastic support during this period. We also hope that you, the readers, have enjoyed 1997 as much as we have, and we look forward to your continued support and encouragement in the coming years.

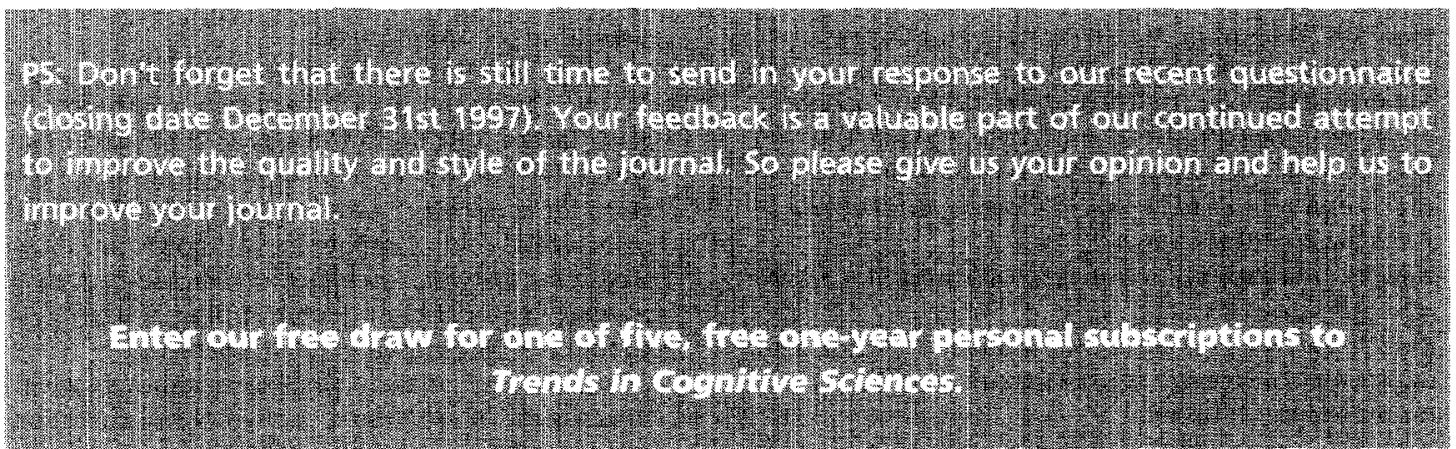

Peter Collins, Editor; Julian Ogilvie, Assistant Editor; Margaret Newell, Production Coordinator; Kevin Halligan, Editorial Administrator. 